typeset using JPSJ.sty $<$ ver.0.8 $>$

\title{
Ground-State and Thermodynamic Properties of the Quantum Mixed Spin-1/2-1/2-1-1 Chain
}

\author{
Takashi Tonegawa \\ Seiji MiYashita $^{3}$ and Hans-Jürgen MikeskA ${ }^{4}$ \\ Department of Physics, Faculty of Science, Kobe University, Rokkodai, Kobe 657 \\ ${ }^{1}$ Division of Information and Media Science, Graduate School of Science and Technology, Kobe University, \\ Rokkodai, Kobe 657 \\ ${ }^{2}$ Department of Informatics, Faculty of Cross-Cultural Studies, Kobe University, Tsurukabuto, Kobe 657 \\ ${ }^{3}$ Department of Earth and Space Science, Graduate School of Science, Osaka University, Toyonaka, Osaka 560 \\ ${ }^{4}$ Institut für Theoretische Physik, Universität Hannover, 30167 Hannover, Germany
}

(Received October 6, 1997)

We investigate both analytically and numerically the ground-state and thermodynamic properties of the quantum mixed spin-1/2-1/2-1-1 chain described by the Hamiltonian $\mathcal{H}=\sum_{\ell=1}^{N / 4}\left(J_{1} \vec{s}_{4 \ell-3} \cdot \vec{s}_{4 \ell-2}+J_{2} \vec{s}_{4 \ell-2} \cdot \vec{S}_{4 \ell-1}+J_{3} \vec{S}_{4 \ell-1} \cdot \vec{S}_{4 \ell}+J_{2} \vec{S}_{4 \ell} \cdot \vec{s}_{4 \ell+1}\right)$, where two $S=1 / 2$ spins $\left(\vec{s}_{4 \ell-3}\right.$ and $\left.\vec{s}_{4 \ell-2}\right)$ and two $S=1$ spins $\left(\vec{S}_{4 \ell-1}\right.$ and $\left.\vec{S}_{4 \ell}\right)$ are arranged alternatively. In several limiting cases of $J_{1}, J_{2}$, and $J_{3}$ we apply the Wigner-Eckart theorem and carry out a perturbation calculation to examine the behavior of the massless lines where the energy gap vanishes. Performing a quantum Monte Carlo calculation without global flips at a sufficiently low temperature for the case where $J_{1}=J_{3}=1.0$ and $J_{2}>0$, we find that the ground state of the present system in this case undergoes a second-order phase transition accompanying the vanishing of the energy gap at $J_{2}=J_{2 \mathrm{c}}$ with $J_{2 \mathrm{c}}=0.77 \pm 0.01$. We also find that the ground states for both $J_{2}<J_{2 \mathrm{c}}$ and $J_{2}>J_{2 \mathrm{c}}$ can be understood by means of the valence-bond-solid picture. A quantum Monte Carlo calculation which takes the global flips along the Trotter direction into account is carried out to elucidate the temperature dependences of the specific heat and the magnetic susceptibility. In particular, it is found that the susceptibility per unit cell for $J_{2}=0.77$ with $J_{1}=J_{3}=1.0$ takes a finite value at absolute zero temperature and that the specific heat per unit cell versus temperature curve for $J_{2}=5.0$ with $J_{1}=J_{3}=1.0$ has a double peak.

KEYWORDS: quantum mixed spin-1/2-1/2-1-1 chain, ground-state properties, thermodynamic properties, quantum Monte Carlo calculation, ground-state phase transition, specific heat, magnetic susceptibility

\footnotetext{
* tonegawa@kobe-u.ac.jp

** hikihara@phys560.phys.kobe-u.ac.jp
} 


\section{$\S 1$. Introduction}

A quantum antiferromagnetic chain has been the subject of numerous experimental as well as theoretical studies over a long period, in particular since Haldane's predictionl implying that the chain with an integer spin is quite different from the chain with a half-integer spin in the ground-state and low-lying-excited-state properties. Almost all results of these studies support the prediction, and now it is widely agreed that for the case of isotropic nearest-neighbor exchange interactions, the former chain has massive excitations with a exponential decay of the two-spin correlation functions, while the latter chain has massless excitations with a power-law decay of the two-spin correlation functions.2)

Under these circumstances, a mixed spin chain consisting of two kinds of quantum spins which are arranged periodically has been of considerable interest in recent years. On the experimental side, several real materials of this type have been synthesized, and their magnetic properties have been observed.3) A typical example of these materials is $\mathrm{CuNi}(\mathrm{pba})\left(\mathrm{H}_{2} \mathrm{O}\right)_{3} \cdot 2 \mathrm{H}_{2} \mathrm{O}$ with pba=1,3propylenebis(oxamato), 1 where an $S=1 / 2$ spin of the $\mathrm{Cu}^{2+}$ ion and an $S=1$ spin of the $\mathrm{Ni}^{2+}$ ion make an alternating array and each nearest-neighbor pair of the spins couples by the isotropic, antiferromagnetic exchange interaction. On the theoretical side, on the other hand, a variety of models

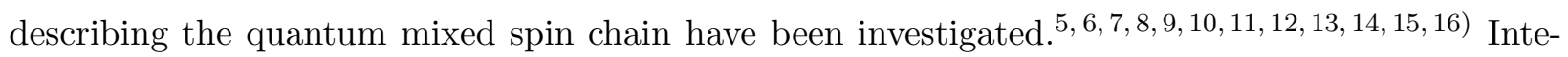
grable models have been constructed and discussed by several authors. 6. 6.8 Although these integrable models are specific ones in which rather complicated interactions are assumed, exact solutions for the models are helpful for us to understand the essential consequences of the quantum mixed spin chain. Motivated by the above experimental observations, several authors, (10, 14, 12, 14, 15, (6) have studied both analytically and numerically a simpler case of the $S=1 / 2$ and $S=1$ alternating spin chain with antiferromagnetic nearest-neighbor exchange interactions. A characteristic feature of this chain in the case of isotropic or Ising-type interactions is that it is a quantum ferrimagnet and its ground state is magnetic.

Generally speaking, quantum fluctuations play a more crucial role in the nonmagnetic ground state than in the magnetic one. As an example of the quantum mixed spin chains which have isotropic nearest-neighbor exchange interactions only and also have a nonmagnetic ground state, we consider in this paper a one-dimensional antiferromagnet where two $S=1 / 2$ and two $S=1$ spins are arranged alternatively. For the sake of simplicity we call this system the spin-1/2-1/2-1-1 chain. The Hamiltonian is given by

$$
\mathcal{H}=\sum_{\ell=1}^{N / 4}\left(J_{1} \vec{s}_{4 \ell-3} \cdot \vec{s}_{4 \ell-2}+J_{2} \vec{s}_{4 \ell-2} \cdot \vec{S}_{4 \ell-1}+J_{3} \vec{S}_{4 \ell-1} \cdot \vec{S}_{4 \ell}+J_{2} \vec{S}_{4 \ell} \cdot \vec{s}_{4 \ell+1}\right),
$$

where $\vec{s}_{\ell}$ with $\ell=1$ and $2(\bmod 4)$ and $\vec{S}_{\ell}$ with $\ell=3$ and $4(\bmod 4)$ are, respectively, the $S=1 / 2$ and $S=1$ spin operators at the $\ell$ th site; $J_{1}, J_{2}$, and $J_{3}$ are, respectively, the interaction constants 
between the nearest-neighbor pair of $S=1 / 2$ spins, that of $S=1 / 2$ and $S=1$ spins, and that of $S=1$ spins; $N$, being assumed to be a multiple of four, is the total number of spins. We impose periodic boundary conditions $\left(\vec{s}_{N+1} \equiv \vec{s}_{1}\right)$.

It is naturally anticipated that the present mixed spin system has different ground states depending upon the signs of $J_{1}, J_{2}$, and $J_{3}$. As we will discuss later in more detail (see Table I below), the value $\mathcal{S}_{\text {tot }}$ of the total spin

$$
\overrightarrow{\mathcal{S}}_{\text {tot }}=\sum_{\ell=1}^{N / 4}\left\{\vec{s}_{4 \ell-3}+\vec{s}_{4 \ell-2}+\vec{S}_{4 \ell-1}+\vec{S}_{4 \ell}\right\}
$$

in the ground state can be determined rigorously by applying the Lieb-Mattis theorem.17) Except in the region where $J_{1}<0$ and $J_{3}<0$, the ground state belongs to the $\mathcal{S}_{\text {tot }}=0$ subspace, and therefore it is nonmagnetic. Furthermore, in the region where $J_{1}<0, J_{2}>0$, and $J_{3}<0$ and in the region where $J_{1}<0, J_{2}<0$, and $J_{3}<0$, the ground states belong, respectively, to the $\mathcal{S}_{\text {tot }}=N / 4$ and $\mathcal{S}_{\text {tot }}=3 N / 4$ subspaces. The former is ferrimagnetic, while the latter is ferromagnetic. For comparison we depict in Fig. 1 the spin array with the lowest energy of the corresponding classical system in which $\vec{s}_{\ell}$ and $\vec{S}_{\ell}$ are replaced, respectively, by the classical spin vectors whose magnitudes are $1 / 2$ and 1 . From this figure and Table I we find that there is a close analogy between the classical lowest-energy state and the quantum ground state. It is interesting to see that the periods concerning the translational symmetry of the classical lowest-energy states are, respectively, four and eight in units of the lattice spacing in the region where $J_{1} J_{3}>0$ and in the region where $J_{1} J_{3}<0$.

We explore here both analytically and numerically the ground-state and thermodynamic properties of the present system 18 In several limiting cases of the interaction constants we apply the Wigner-Eckart theorem 19 ) and carry out a perturbation calculation to examine the behavior of the massless lines in the $J_{1}$ versus $J_{2}$ plane with $J_{1}>0$ and $J_{3}>0$. Confining ourselves to the case where $J_{1}=J_{3}=1.0$ and $J_{2}>0$, we perform numerical calculations. In these calculations we mainly employ a quantum Monte Carlo (QMC) method, and do an exact-diagonalization calculation for $N=8$ and 16 to check the QMC results. To determine as accurately as possible the massless point $J_{2 \mathrm{c}}$ of $J_{2}$ in the above case is one of our main purposes. We discuss how the ground states for both $J_{2}<J_{2 \mathrm{c}}$ and $J_{2}>J_{2 \mathrm{c}}$ are represented in the frame of the valence-bond-solid (VBS) picture proposed by Affleck, Kennedy, Lieb, and Tasaki 20) We also aim at clarifying the temperature dependences of the specific heat and the magnetic susceptibility for a few values of $J_{2}$ in this case. Very recently, Fukui and Kawakami14 have also discussed this system. They have mapped this system to the non-linear sigma model and have shown that in its ground state a second-order phase transition which accompanies the vanishing of the energy gap may occur.

The remainder of the paper is organized as follows. The next section ( $\$ 2$ ) and the following one $(\S 3)$ are devoted to analytical treatments and numerical calculations, respectively. Finally, the main results are summarized and further discussed in $\S 4$. 


\section{§2. Analytical Treatments}

We start with applying the Lieb-Mattis theorem 17 ) to determine the value $\mathcal{S}_{\text {tot }}$ of the total spin $\overrightarrow{\mathcal{S}}_{\text {tot }}$ in the ground state for arbitrary values of $J_{1}, J_{2}$, and $J_{3}$. In the present case the theorem implies that, if we can divide the lattice into the $A$ and $B$ sublattices in such a way that all the intrasublattice interaction constants are not positive and all the intersublattice interaction constants are not negative, then $\mathcal{S}_{\text {tot }}$ is given by

$$
\mathcal{S}_{\text {tot }}=\left|\mathcal{S}_{\max , A}-\mathcal{S}_{\max , B}\right|
$$

where $\mathcal{S}_{\max , A}$ and $\mathcal{S}_{\max , B}$ are, respectively, the maximum values of the total spins in the $A$ and $B$ sublattices. (Note that the constant $g^{2}$ in ref. 17 is equal to zero because the Hamiltonian $\mathcal{H}$ consists of the nearest-neighbor interactions only.) Let us consider two examples. For the region where $J_{1}>0, J_{2}>0$, and $J_{3}>0$, we choose the sites $\ell_{A}$ belonging to the $A$ sublattice to be $\ell_{A}=1$ $(\bmod 2)$ and the sites $\ell_{B}$ belonging to the $B$ sublattice to be $\ell_{B}=2(\bmod 2)$. Then, all of $J_{1}, J_{2}$, and $J_{3}$ are the intersublattice interaction constants, which satisfy the above requirement, and thus we obtain $\mathcal{S}_{\text {tot }}=0$ since $\mathcal{S}_{\max , A}=\mathcal{S}_{\max , B}=3 N / 8$. For the region where $J_{1}>0, J_{2}<0$, and $J_{3}>0$, on the other hand, we choose $\ell_{A}$ to be $\ell_{A}=1$ and $4(\bmod 4)$ and $\ell_{B}$ to be $\ell_{B}=2$ and $3(\bmod 4)$. Then, $J_{2}$ is the intrasublattice interaction constant and the remaining $J_{1}$ and $J_{3}$ are the intersublattice interaction constants, which again satisfy the above requirement. Thus, we obtain again $\mathcal{S}_{\text {tot }}=0$ since $\mathcal{S}_{\max , A}=\mathcal{S}_{\max , B}=3 N / 8$. In a similar way, we can determine $\mathcal{S}_{\text {tot }}$ for the remaining regions of $J_{1}, J_{2}$, and $J_{3}$. The results are listed in Table I together with $\ell_{A}, \ell_{B}, \mathcal{S}_{\max , A}$, and $\mathcal{S}_{\max , B}$ for each region. It should be noted that the $A$ and $B$ sublattices correspond, respectively, to the 'up' and 'down' sublattices in the lowest-energy classical-spin arrays shown in Fig. 1.

We now turn to an application of the Wigner-Eckart theorem 19) to the following three limiting cases: (a) the case where $\left|J_{2}\right| \gg\left|J_{1}\right|,\left|J_{3}\right|$ with $J_{2}>0$, (b) the case where $\left|J_{2}\right| \gg\left|J_{1}\right|,\left|J_{3}\right|$ with $J_{2}<0$, and (c) the case where $\left|J_{1}\right| \gg\left|J_{2}\right|,\left|J_{3}\right|$ with $J_{1}<0$. In the limiting case (a), a pair of the spins $\vec{s}_{4 \ell-2}$ and $\vec{S}_{4 \ell-1}$ can be replaced by an effective $S=1 / 2 \operatorname{spin} \vec{s}_{4 \ell-2,4 \ell-1}^{\text {eff }}$. This replacement means that we take into account only the doublet state of the two-spin system consisting of $\vec{s}_{4 \ell-2}$ and $\vec{S}_{4 \ell-1}$ and neglect the quartet state of the system. Similarly, a pair of the spins $\vec{S}_{4 \ell}$ and $\vec{s}_{4 \ell+1}$ can be replaced by an effective $S=1 / 2$ spin $\vec{s}_{4 \ell, 4 \ell+1}^{\text {eff }}$ Then, the Wigner-Eckart theorem implies that both $\vec{s}_{4 \ell-2}$ and $\vec{S}_{4 \ell-1}$ are proportional to $\vec{s}_{4 \ell-2,4 \ell-1}^{\mathrm{eff}}$ and both $\vec{S}_{4 \ell}$ and $\vec{s}_{4 \ell+1}$ are proportional to $\vec{s}_{4 \ell, 4 \ell+1}^{\text {eff }}$ :

$$
\begin{array}{r}
\vec{s}_{4 \ell-2}=\alpha \vec{s}_{4 \ell-2,4 \ell-1}^{\mathrm{eff}}, \\
\vec{S}_{4 \ell-1}=\beta \vec{s}_{4 \ell-2,4 \ell-1}^{\mathrm{eff}}, \\
\vec{S}_{4 \ell}=\beta^{\prime} \vec{s}_{4 \ell, 4 \ell+1}^{\mathrm{eff}}, \\
\vec{s}_{4 \ell+1}=\alpha^{\prime} \vec{s}_{4 \ell, 4 \ell+1}^{\mathrm{eff}},
\end{array}
$$


Table I. Values of $\ell_{A}, \ell_{B}, \mathcal{S}_{\max , A}, \mathcal{S}_{\max , B}$, and $\mathcal{S}_{\text {tot }}$ for each region of $J_{1}, J_{2}$, and $J_{3}$.

\begin{tabular}{|c|c|c|c|c|c|}
\hline Region of $J_{1}, J_{2}$, and $J_{3}$ & $\ell_{A}$ & $\ell_{B}$ & $\mathcal{S}_{\max , A}$ & $\mathcal{S}_{\max , B}$ & $\mathcal{S}_{\text {tot }}$ \\
\hline$J_{1}>0, J_{2}>0, J_{3}>0$ & $1(\bmod 2)$ & $2(\bmod 2)$ & $3 N / 8$ & $3 N / 8$ & 0 \\
\hline$J_{1}>0, J_{2}<0, J_{3}>0$ & $1,4(\bmod 4)$ & $2,4(\bmod 4)$ & $3 N / 8$ & $3 N / 8$ & 0 \\
\hline$J_{1}<0, J_{2}<0, J_{3}>0$ & $1,2,3,8(\bmod 8)$ & $4,5,6,7(\bmod 8)$ & $3 N / 8$ & $3 N / 8$ & 0 \\
\hline$J_{1}<0, J_{2}>0, J_{3}>0$ & $1,2,4,7(\bmod 8)$ & $3,5,6,8(\bmod 8)$ & $3 N / 8$ & $3 N / 8$ & 0 \\
\hline$J_{1}>0, J_{2}>0, J_{3}<0$ & $1,3,4,6(\bmod 8)$ & $2,5,7,8(\bmod 8)$ & $3 N / 8$ & $3 N / 8$ & 0 \\
\hline$J_{1}>0, J_{2}<0, J_{3}<0$ & $1,6,7,8(\bmod 8)$ & $2,3,4,5(\bmod 8)$ & $3 N / 8$ & $3 N / 8$ & 0 \\
\hline$J_{1}<0, J_{2}<0, J_{3}<0$ & all & none & $3 N / 4$ & 0 & $3 N / 4$ \\
\hline$J_{1}<0, J_{2}>0, J_{3}<0$ & $1,2(\bmod 4)$ & $3,4(\bmod 4)$ & $N / 4$ & $N / 2$ & $N / 4$ \\
\hline
\end{tabular}

$\alpha, \beta, \beta^{\prime}$, and $\alpha^{\prime}$ being constants. The values of $\alpha, \beta, \beta^{\prime}$, and $\alpha^{\prime}$ can be obtained, respectively, by calculating all matrix elements of both sides of eq. (2.2a), eq. (2.2b), eq. (2.2c), and eq. (2.2d) in the doublet subspace. The results are $\alpha=\alpha^{\prime}=-1 / 3$ and $\beta=\beta^{\prime}=4 / 3$. Substituting eqs. (2.2a)(2.2d) with these constants into eq. (1.1), we obtain, apart from the constant term coming from the $J_{2}$-terms,

$$
\mathcal{H}_{(\mathrm{a})}=\sum_{\ell=1}^{N / 4}\left(\frac{1}{9} J_{1} \vec{s}_{4 \ell-4,4 \ell-3}^{\mathrm{eff}} \cdot \vec{s}_{4 \ell-2,4 \ell-1}^{\mathrm{eff}}+\frac{16}{9} J_{3} \vec{s}_{4 \ell-2,4 \ell-1}^{\mathrm{eff}} \cdot \vec{s}_{4 \ell, 4 \ell+1}^{\mathrm{eff}}\right)
$$

with $\vec{s}_{0,1}^{\text {eff }} \equiv \vec{s}_{N, N+1}^{\text {eff }} \equiv \vec{s}_{N, 1}^{\text {eff }}$. Thus, we can conclude that in the limiting case (a), our system described by the Hamiltonian $\mathcal{H}$ of eq. (1.1) is equivalent to the $S=1 / 2$ bond-alternating chain described by the Hamiltonian $\mathcal{H}_{(\text {a) }}$ of eq. (2.3), as far as the ground state and the sufficiently low-energy excited states are concerned.

In the limiting case (b), we replace a pair of the spins $\vec{s}_{4 \ell-2}$ and $\vec{S}_{4 \ell-1}\left(\vec{S}_{4 \ell}\right.$ and $\left.\vec{s}_{4 \ell+1}\right)$ by an effective $S=3 / 2$ spin $\vec{\Sigma}_{4 \ell-2,4 \ell-1}^{\text {eff }}\left(\vec{\Sigma}_{4 \ell, 4 \ell+1}^{\text {eff }}\right)$, neglecting the doublet state of the two-spin system consisting of $\vec{s}_{4 \ell-2}$ and $\vec{S}_{4 \ell-1}\left(\vec{S}_{4 \ell}\right.$ and $\left.\vec{s}_{4 \ell+1}\right)$. Applying the Wigner-Eckart theorem, we obtain

$$
\begin{array}{r}
\vec{s}_{4 \ell-2}=\frac{1}{3} \vec{\Sigma}_{4 \ell-2,4 \ell-1}^{\mathrm{eff}}, \\
\vec{S}_{4 \ell-1}=\frac{2}{3} \vec{\Sigma}_{4 \ell-2,4 \ell-1}^{\mathrm{eff}}, \\
\vec{S}_{4 \ell}=\frac{2}{3} \vec{\Sigma}_{4 \ell, 4 \ell+1}^{\mathrm{eff}}, \\
\vec{s}_{4 \ell+1}=\frac{1}{3} \vec{\Sigma}_{4 \ell, 4 \ell+1}^{\mathrm{eff}} .
\end{array}
$$


Thus, we can show that in the limiting case (b), our system is equivalent to the $S=3 / 2$ bondalternating chain described by the following Hamiltonian:

$$
\mathcal{H}_{(\mathrm{b})}=\sum_{\ell=1}^{N / 4}\left(\frac{1}{9} J_{1} \vec{\Sigma}_{4 \ell-4,4 \ell-3}^{\mathrm{eff}} \cdot \vec{\Sigma}_{4 \ell-2,4 \ell-1}^{\mathrm{eff}}+\frac{4}{9} J_{3} \vec{\Sigma}_{4 \ell-2,4 \ell-1}^{\mathrm{eff}} \cdot \vec{\Sigma}_{4 \ell, 4 \ell+1}^{\mathrm{eff}}\right)
$$

with $\vec{\Sigma}_{0,1}^{\text {eff }} \equiv \vec{\Sigma}_{N, N+1}^{\text {eff }} \equiv \vec{\Sigma}_{N, 1}^{\text {eff }}$, as far as the ground state and the sufficiently low-energy excited states are concerned. Furthermore, in the limiting case (c), we replace a pair of the spins $\vec{s}_{4 \ell-3}$ and $\vec{s}_{4 \ell-2}$ by an effective $S=1$ spin $\vec{S}_{4 \ell-3,4 \ell-2}^{\text {eff }}$, neglecting the singlet state of the two-spin system consisting of $\vec{s}_{4 \ell-3}$ and $\vec{s}_{4 \ell-2}$. The Wigner-Eckart theorem gives

$$
\begin{aligned}
& \vec{s}_{4 \ell-3}=\frac{1}{2} \vec{S}_{4 \ell-3,4 \ell-2}^{\mathrm{eff}}, \\
& \vec{s}_{4 \ell-2}=\frac{1}{2} \vec{S}_{4 \ell-3,4 \ell-2}^{\mathrm{eff}} .
\end{aligned}
$$

Thus, our system in the limiting case (c) is equivalent to the $S=1$ chain described by the following Hamiltonian:

$$
\mathcal{H}_{(\mathrm{c})}=\sum_{\ell=1}^{N / 4}\left(\frac{1}{2} J_{2} \vec{S}_{4 \ell-3,4 \ell-2}^{\mathrm{eff}} \cdot \vec{S}_{4 \ell-1}+J_{3} \vec{S}_{4 \ell-1} \cdot \vec{S}_{4 \ell}+\frac{1}{2} J_{2} \vec{S}_{4 \ell} \cdot \vec{S}_{4 \ell+1,4 \ell+2}^{\mathrm{eff}}\right)
$$

with $\vec{S}_{N+1, N+2}^{\text {eff }} \equiv \vec{S}_{1,2}^{\text {eff }}$, as far as the ground state and the sufficiently low-energy excited states are concerned.

From the above arguments for the first two limiting cases (a) and (b), we can discuss when the system has massless excitations in these cases. In the case (a), it is massless only when

$$
J_{1}=16 J_{3}>0
$$

since the antiferromagnetic $S=1 / 2$ bond-alternating chain becomes massless only in the case of no bond-alternation.21) In the case (b), on the other hand, the system is massless when22, 23)

$$
J_{1}=4 J_{3}>0 \quad \text { and } \quad J_{1}=4 J_{3} \frac{1 \pm \delta}{1 \mp \delta}>0 .
$$

The value of $\delta$ has been determined24.25) recently to be $\delta=0.43 \pm 0.01$, which is the value given in ref. 25.

We also investigate the possibility of massless lines in the limiting cases: (d) the case where $J_{3} \gg\left|J_{1}\right|,\left|J_{2}\right|$ and (e) the case where $J_{1} \gg\left|J_{2}\right|$ and $J_{1} \gg J_{3}>0$. For this purpose we perform perturbation calculations. For the case (d), we first observe that the system is massless at the point $J_{1}=J_{2}=0.0$ when $J_{3}>0$. This is because at this point the system consists of $N / 4$ independent pairs of two $S=1$ spins, each of which has a singlet state as the lowest-energy state, plus $N / 2$ free $S=1 / 2$ spins, and therefore the ground state of the system is $2^{N / 2}$-fold degenerate. In order to 
explore the neighborhood of the above point for finite $J_{3}(>0)$, we consider the four-spin system described by the Hamiltonian,

$$
h_{\ell}^{(4)}=J_{2} \vec{s}_{4 \ell-2} \cdot \vec{S}_{4 \ell-1}+J_{3} \vec{S}_{4 \ell-1} \cdot \vec{S}_{4 \ell}+J_{2} \vec{S}_{4 \ell} \cdot \vec{s}_{4 \ell+1}
$$

Diagonalizing analytically this Hamiltonian, we find that the ground and first-excited states of the four-spin system are singlet and triplet states, respectively. The energy difference $\tilde{J}$ between them is given by

$$
\tilde{J}=J_{2}\left\{\frac{4}{3}\left(\frac{J_{2}}{J_{3}}\right)+2\left(\frac{J_{2}}{J_{3}}\right)^{2}-\frac{47}{27}\left(\frac{J_{2}}{J_{3}}\right)^{3}+O\left(\left(\frac{J_{2}}{J_{3}}\right)^{4}\right)\right\},
$$

while the energies of the second- and higher-excited states measured from the ground-state energy are finite at $J_{2}=0.0$. Thus, taking only the ground and first-excited states into consideration, we regard the four-spin system as the system described by $\tilde{J} \vec{\sigma}_{\ell} \cdot \vec{\tau}_{\ell}$, where both $\vec{\sigma}_{\ell}$ and $\vec{\tau}_{\ell}$ are $S=1 / 2$ operators. Then, as far as the ground state and the sufficiently low-energy excited states are concerned, we map our original system described by the Hamiltonian $\mathcal{H}$ of eq. (1.1) to the following effective Hamiltonian:

$$
\mathcal{H}_{\mathrm{eff}}=\sum_{\ell=1}^{N / 4}\left(\tilde{J} \vec{\sigma}_{\ell} \cdot \vec{\tau}_{\ell}+\tilde{J}_{1} \vec{\tau}_{\ell} \cdot \vec{\sigma}_{\ell+1}+\tilde{J}_{2} \vec{\sigma}_{\ell} \cdot \vec{\sigma}_{\ell+1}+\tilde{J}_{2} \vec{\tau}_{\ell} \cdot \vec{\tau}_{\ell+1}\right)
$$

with $\vec{\sigma}_{\frac{N}{4}+1} \equiv \vec{\sigma}_{1}$ and $\vec{\tau}_{\frac{N}{4}+1} \equiv \vec{\tau}_{1}$. The values of $\tilde{J}_{1}$ and $\tilde{J}_{2}$ are determined in such a way that the matrix elements of the two operators $\tilde{J}_{1} \vec{\tau}_{\ell} \cdot \vec{\sigma}_{\ell+1}+\tilde{J}_{2} \vec{\sigma}_{\ell} \cdot \vec{\sigma}_{\ell+1}+\tilde{J}_{2} \vec{\tau}_{\ell} \cdot \vec{\tau}_{\ell+1}$ and $J_{1} \vec{s}_{4 \ell+1} \cdot \vec{s}_{4 \ell+2}$ coincide. The mapping is correct to the order of $\left(J_{2} / J_{3}\right)^{3}$, and $\tilde{J}_{1}$ and $\tilde{J}_{2}$ are given by

$$
\begin{aligned}
& \tilde{J}_{1}=J_{1}\left\{1-\frac{4}{3}\left(\frac{J_{2}}{J_{3}}\right)^{2}-\frac{4}{3}\left(\frac{J_{2}}{J_{3}}\right)^{3}+O\left(\left(\frac{J_{2}}{J_{3}}\right)^{4}\right)\right\}, \\
& \tilde{J}_{2}=J_{1}\left\{\frac{2}{3}\left(\frac{J_{2}}{J_{3}}\right)^{3}+O\left(\left(\frac{J_{2}}{J_{3}}\right)^{4}\right)\right\} .
\end{aligned}
$$

Thus, to the order of $\left(J_{2} / J_{3}\right)^{3}$, the effective Hamiltonian $\mathcal{H}_{\text {eff }}$ describes the $S=1 / 2$ chain which has the bond-alternating nearest-neighbor interactions with the interaction constants $\tilde{J}$ and $\tilde{J}_{1}$ and also the uniform next-nearest-neighbor interaction with the interaction constant $\tilde{J}_{2}$. Thus, remembering the fact that the antiferromagnetic $S=1 / 2$ chain with the uniform nearest-neighbor and next-nearest-neighbor interactions where the ratio of the latter interaction to the former one is less than 0.24 is massless, 26) we may conclude, from eqs. (2.11), (2.13a), and (2.13b), that in 
the limiting case (d), the present quantum mixed spin chain described by the Hamiltonian $\mathcal{H}$ of eq. (1.1) is massless when

$$
J_{1}=J_{2}\left\{\frac{4}{3}\left(\frac{J_{2}}{J_{3}}\right)+2\left(\frac{J_{2}}{J_{3}}\right)^{2}+\frac{1}{27}\left(\frac{J_{2}}{J_{3}}\right)^{3}+O\left(\left(\frac{J_{2}}{J_{3}}\right)^{4}\right)\right\} .
$$

For the case (e) we perform a similar perturbation calculation in the following way. We first diagonalize analytically the Hamiltonian which describes another four-spin system,

$$
h_{\ell}^{(4)}=J_{2} \vec{S}_{4 \ell-4} \cdot \vec{s}_{4 \ell-3}+J_{1} \vec{s}_{4 \ell-3} \cdot \vec{s}_{4 \ell-2}+J_{2} \vec{s}_{4 \ell-2} \cdot \vec{S}_{4 \ell-1},
$$

to obtain the energy difference $\tilde{J}$ between the singlet ground and triplet first-excited states and that $\tilde{J}^{\prime}$ between the singlet ground and quintet second-excited state to be

$$
\begin{gathered}
\tilde{J}=J_{1}\left\{\frac{1}{2}\left(\frac{J_{2}}{J_{1}}\right)^{2}+O\left(\left(\frac{J_{2}}{J_{1}}\right)^{3}\right)\right\}, \\
\tilde{J}^{\prime}=J_{1}\left\{\frac{3}{2}\left(\frac{J_{2}}{J_{1}}\right)^{2}+O\left(\left(\frac{J_{2}}{J_{1}}\right)^{3}\right)\right\} .
\end{gathered}
$$

Adding the fact that the energy difference between these lowest states and higher-excited states are finite at $\left|J_{2}\right| / J_{1}=0.0$, we can regard, to the order of $\left(J_{2} / J_{1}\right)^{2}$, the four-spin system as a system described by $\tilde{J}_{\ell} \cdot \vec{\tau}_{\ell}$, where $\vec{\sigma}_{\ell}$ and $\vec{\tau}_{\ell}$ are $S=1$ operators. Then, as far as the ground state and the sufficiently low-energy excited states are concerned, we map the present system described by the Hamiltonian $\mathcal{H}$ of eq. (1.1) to the effective Hamiltonian of eq. (2.12) with the $S=1$ operators $\vec{\sigma}_{\ell}$ and $\vec{\tau}_{\ell}$. The values of $\tilde{J}_{1}$ and $\tilde{J}_{2}$ can be determined similarly to the above perturbation calculation. The results are given by

$$
\begin{aligned}
& \tilde{J}_{1}=J_{3}\left\{1-\frac{1}{2}\left(\frac{J_{2}}{J_{1}}\right)^{2}+O\left(\left(\frac{J_{2}}{J_{1}}\right)^{3}\right)\right\}, \\
& \tilde{J}_{2}=J_{3} O\left(\left(\frac{J_{2}}{J_{1}}\right)^{3}\right),
\end{aligned}
$$

which show that, to the order of $\left(J_{2} / J_{1}\right)^{2}$, the effective Hamiltonian $\mathcal{H}_{\text {eff }}$ describes the $S=1$ chain which has the bond-alternating nearest-neighbor interactions with the interaction constants $\tilde{J}$ and $\tilde{J}_{1}$. Thus, it may be concluded, from eqs. (2.16a) and (2.17a), that in the limiting case (e), the present system is massless when $\left(J_{1} / 2\right)\left(J_{2} / J_{1}\right)^{2} / J_{3}\left\{1-(1 / 2)\left(J_{2} / J_{1}\right)^{2}\right\}=(1 \pm \delta) /(1 \mp \delta)$ with $\delta \simeq 1 / 4,25,27,28,29,30)$ or equivalently, when

$$
J_{1}=\frac{3}{10} \frac{J_{2}^{2}}{J_{3}} \quad \text { and } \quad J_{1}=\frac{5}{6} \frac{J_{2}^{2}}{J_{3}} .
$$


The above results given by eqs. (2.8), (2.9), (2.14), and (2.18) with $J_{3}$ fixed at a positive and finite value describe, respectively, the asymptotic behavior in the limiting cases (a), (b), (d), and (e) of the massless lines on the $J_{1}$ versus $J_{2}$ plane. These are summarized in Fig. 2, where $J_{3}$ is chosen to be $J_{3}=1.0$. Our preliminary results indicate that the asymptotic massless lines in Fig. 2 connect to form four massless lines. Leaving the detailed presentation of these massless lines for further publication,31 we determine in the next section the massless point of $J_{2}$ for the case where $J_{1}=J_{3}=1.0$ and $J_{2}>0$, as mentioned in $\S 1$.

\section{$\S 3 . \quad$ Numerical calculations}

\subsection{QMC method}

As has been mentioned in $\S 1$, we mainly employ a QMC method in the numerical calculation. Before discussing the numerical results, we explain the QMC procedure for the present spin-1/2 1/2-1-1 chain. By the use of the Suzuki-Trotter decomposition 32 ) of checker-board type,33 the partition function $Z=\operatorname{Tr}[\exp (-\beta \mathcal{H})]$, where $\beta=\left(k_{\mathrm{B}} T\right)^{-1}$ with the Boltzmann constant $k_{\mathrm{B}}$ and the absolute temperature $T$, is approximated as

$$
\begin{aligned}
Z \simeq \operatorname{Tr}\left[\prod_{\ell=1}^{N / 4}\left\{\exp \left(-\beta h_{4 \ell-3,4 \ell-2} / n\right) \exp \left(-\beta h_{4 \ell-1,4 \ell} / n\right)\right\}^{n}\right. \\
\left.\quad \times \prod_{\ell=1}^{N / 4}\left\{\exp \left(-\beta h_{4 \ell-2,4 \ell-1} / n\right) \exp \left(-\beta h_{4 \ell, 4 \ell+1} / n\right)\right\}^{n}\right]
\end{aligned}
$$

Here,

$$
\begin{array}{r}
h_{4 \ell-3,4 \ell-2}=J_{1} \vec{s}_{4 \ell-3} \cdot \vec{s}_{4 \ell-2}, \\
h_{4 \ell-1,4 \ell}=J_{3} \vec{S}_{4 \ell-1} \cdot \vec{S}_{4 \ell}, \\
h_{4 \ell-2,4 \ell-1}=J_{2} \vec{s}_{4 \ell-2} \cdot \vec{S}_{4 \ell-1}, \\
h_{4 \ell, 4 \ell+1}=J_{2} \vec{S}_{4 \ell} \cdot \vec{s}_{4 \ell+1}
\end{array}
$$

are the local Hamiltonians, and $n$ is the Trotter number. Choosing the $z$-axis as the quantization axis, we introduce the local Boltzmann factors defined by

$$
\begin{gathered}
\rho_{4 \ell-3,4 \ell-2}^{(2 r-1,2 r)}=\left\langle s_{4 \ell-3}^{(2 r-1)} s_{4 \ell-2}^{(2 r-1)}\left|\exp \left(-\beta h_{4 \ell-3,4 \ell-2} / n\right)\right| s_{4 \ell-3}^{(2 r)} s_{4 \ell-2}^{(2 r)}\right\rangle \\
\rho_{4 \ell-1,4 \ell}^{(2 r-1,2 r)}=\left\langle S_{4 \ell-1}^{(2 r-1)} S_{4 \ell}^{(2 r-1)}\left|\exp \left(-\beta h_{4 \ell-1,4 \ell} / n\right)\right| S_{4 \ell-1}^{(2 r)} S_{4 \ell}^{(2 r)}\right\rangle, \\
\rho_{4 \ell-2,4 \ell-1}^{(2 r, 2 r+1)}=\left\langle s_{4 \ell-2}^{(2 r)} S_{4 \ell-1}^{(2 r)}\left|\exp \left(-\beta h_{4 \ell-2,4 \ell-1} / n\right)\right| s_{4 \ell-2}^{(2 r+1)} S_{4 \ell-1}^{(2 r+1)}\right\rangle,
\end{gathered}
$$




$$
\rho_{4 \ell, 4 \ell+1}^{(2 r, 2 r+1)}=\left\langle S_{4 \ell}^{(2 r)} s_{4 \ell+1}^{(2 r)}\left|\exp \left(-\beta h_{4 \ell, 4 \ell+1} / n\right)\right| S_{4 \ell}^{(2 r+1)} s_{4 \ell+1}^{(2 r+1)}\right\rangle
$$

with $\left|s_{4 \ell-3}^{\left(r^{\prime}\right)} s_{4 \ell-2}^{\left(r^{\prime}\right)}\right\rangle,\left|S_{4 \ell-1}^{\left(r^{\prime}\right)} S_{4 \ell}^{\left(r^{\prime}\right)}\right\rangle,\left|s_{4 \ell-2}^{\left(r^{\prime}\right)} S_{4 \ell-1}^{\left(r^{\prime}\right)}\right\rangle$, and $\left|S_{4 \ell}^{\left(r^{\prime}\right)} s_{4 \ell+1}^{\left(r^{\prime}\right)}\right\rangle$, which denote, respectively, the spin states consisting of $\vec{s}_{4 \ell-3}$ and $\vec{s}_{4 \ell-2}$, of $\vec{S}_{4 \ell-1}$ and $\vec{S}_{4 \ell}$, of $\vec{s}_{4 \ell-2}$ and $\vec{S}_{4 \ell-1}$, and of $\vec{S}_{4 \ell}$ and $\vec{s}_{4 \ell+1}$. Here, $r^{\prime}$ is a label along the Trotter direction, taking the values, $1,2, \cdots, 2 n ; s_{4 \ell-3}^{\left(r^{\prime}\right)}$ and $s_{4 \ell-2}^{\left(r^{\prime}\right)}$ take the values, $\pm \frac{1}{2} ; S_{4 \ell-1}^{\left(r^{\prime}\right)}$ and $S_{4 \ell}^{\left(r^{\prime}\right)}$ take the values, $0, \pm 1$. Using these local Boltzmann factors, eq. (3.1) is rewritten as

$$
Z \simeq \sum_{\left\{s_{4 \ell-3}^{\left(r^{\prime}\right)}\right\}} \sum_{\left\{s_{4 \ell-2}^{\left(r^{\prime}\right)}\right\}} \sum_{\left\{S_{4 \ell-1}^{\left(r^{\prime}\right)}\right\}} \sum_{\left\{S_{4 \ell}^{\left(r^{\prime}\right)}\right\}} \prod_{r=1}^{n}\left\{\prod_{\ell=1}^{N / 4}\left(\rho_{4 \ell-3,4 \ell-2}^{(2 r-1,2 r)} \rho_{4 \ell-1,4 \ell}^{(2 r-1,2 r)}\right) \prod_{\ell=1}^{N / 4}\left(\rho_{4 \ell-2,4 \ell-1}^{(2 r, 2 r+1)} \rho_{4 \ell, 4 \ell+1}^{(2 r, 2 r+1)}\right)\right\}
$$

where $\rho_{4 \ell-2,4 \ell-1}^{(2 n, 2 n+1)} \equiv \rho_{4 \ell-2,4 \ell-1}^{(2 n, 1)}$ and $\rho_{4 \ell, 4 \ell+1}^{(2 n, 2 n+1)} \equiv \rho_{4 \ell, 4 \ell+1}^{(2 n, 1)}$. The right-hand side of eq. (3.4) can be regarded as the partition function of a two-dimensional Ising system with the Ising variables, $\left\{s_{4 \ell-3}^{\left(r^{\prime}\right)}\right\},\left\{s_{4 \ell-2}^{\left(r^{\prime}\right)}\right\},\left\{S_{4 \ell-1}^{\left(r^{\prime}\right)}\right\}$, and $\left\{S_{4 \ell}^{\left(r^{\prime}\right)}\right\}$, which has four-body interactions corresponding to the local Boltzmann factors. A graphical representation of this two-dimensional Ising system is presented in Fig. 3. It is noted that in this figure we have four kinds of plaquettes, corresponding to four kinds of the local Boltzmann factors.

We perform a QMC calculation on the basis of the two-dimensional Ising system discussed above. To update the spin configuration, we carry out the following local flips, keeping the total magnetization of the system constant:34)

$$
\begin{gathered}
\left\{s_{4 \ell-3}^{(2 r)}=\frac{1}{2}, s_{4 \ell-2}^{(2 r)}=-\frac{1}{2}, s_{4 \ell-3}^{(2 r+1)}=\frac{1}{2}, s_{4 \ell-2}^{(2 r+1)}=-\frac{1}{2}\right\} \\
\rightleftarrows\left\{s_{4 \ell-3}^{(2 r)}=-\frac{1}{2}, s_{4 \ell-2}^{(2 r)}=\frac{1}{2}, s_{4 \ell-3}^{(2 r+1)}=-\frac{1}{2}, s_{4 \ell-2}^{(2 r+1)}=\frac{1}{2}\right\}, \\
\left\{s_{4 \ell-2}^{(2 r-1)}=\frac{1}{2}, S_{4 \ell-1}^{(2 r-1)}, s_{4 \ell-2}^{(2 r)}=\frac{1}{2}, S_{4 \ell-1}^{(2 r)}\right\} \\
\rightleftarrows\left\{s_{4 \ell-2}^{(2 r-1)}=-\frac{1}{2}, S_{4 \ell-1}^{(2 r-1)}+1, s_{4 \ell-2}^{(2 r)}=-\frac{1}{2}, S_{4 \ell-1}^{(2 r)}+1\right\}, \\
\left\{S_{4 \ell-1}^{(2 r)}, S_{4 \ell}^{(2 r)}, S_{4 \ell-1}^{(2 r+1)}, S_{4 \ell}^{(2 r+1)}\right\} \\
\rightleftarrows\left\{S_{4 \ell-1}^{(2 r)}+1, S_{4 \ell}^{(2 r)}-1, S_{4 \ell-1}^{(2 r+1)}+1, S_{4 \ell}^{(2 r+1)}-1\right\} \\
\left\{S_{4 \ell-1}^{(2 r)}=1, S_{4 \ell}^{(2 r)}=-1, S_{4 \ell-1}^{(2 r+1)}=1, S_{4 \ell}^{(2 r+1)}=-1\right\}
\end{gathered}
$$




$$
\begin{aligned}
& \rightleftarrows\left\{S_{4 \ell-1}^{(2 r)}=-1, S_{4 \ell}^{(2 r)}=1, S_{4 \ell-1}^{(2 r+1)}=-1, S_{4 \ell}^{(2 r+1)}=1\right\}, \\
\left\{S_{4 \ell}^{(2 r-1)}, s_{4 \ell+1}^{(2 r-1)}=-\frac{1}{2}, S_{4 \ell}^{(2 r)}, s_{4 \ell+1}^{(2 r)}=-\frac{1}{2}\right\} & \rightleftarrows\left\{S_{4 \ell}^{(2 r-1)}-1, s_{4 \ell+1}^{(2 r-1)}=+\frac{1}{2}, S_{4 \ell}^{(2 r)}-1, s_{4 \ell+1}^{(2 r)}=+\frac{1}{2}\right\},
\end{aligned}
$$

$S_{4 \ell-1}^{\left(r^{\prime}\right)}\left(r^{\prime}=2 r-1,2 r, 2 r+1\right)$ in eqs. (3.5b) and (3.5c) being equal to -1 or 0 , and $S_{4 \ell}^{\left(r^{\prime}\right)}\left(r^{\prime}=2 r-1\right.$, $2 r, 2 r+1)$ in eqs. $(3.5 \mathrm{c})$ and $(3.5 \mathrm{e})$ is equal to 0 or 1 .

We also carry out the global flips along the Trotter direction, which are given by 34 )

$$
\begin{aligned}
& \left\{s_{\ell^{\prime}}^{(1)}=\frac{1}{2}, s_{\ell^{\prime}}^{(2)}=\frac{1}{2}, \cdots, s_{\ell^{\prime}}^{(2 n)}=\frac{1}{2}\right\} \\
& \left.\stackrel{\{}{\rightleftarrows} s_{\ell^{\prime}}^{(1)}=-\frac{1}{2}, s_{\ell^{\prime}}^{(2)}=-\frac{1}{2}, \cdots, s_{\ell^{\prime}}^{(2 n)}=-\frac{1}{2}\right\} \quad\left(\ell^{\prime}=4 \ell-3,4 \ell-2\right), \\
& \left\{S_{\ell^{\prime}}^{(1)}, S_{\ell^{\prime}}^{(2)}, \cdots, S_{\ell^{\prime}}^{(2 n)}\right\} \\
& \rightleftarrows\left\{S_{\ell^{\prime}}^{(1)}-1, S_{\ell^{\prime}}^{(2)}-1, \cdots, S_{\ell^{\prime}}^{(2 n)}-1\right\} \quad\left(\ell^{\prime}=4 \ell-1,4 \ell\right),
\end{aligned}
$$

$S_{\ell^{\prime}}^{\left(r^{\prime}\right)}\left(r^{\prime}=1,2, \cdots, 2 n\right)$ in eq. $(3.6 \mathrm{~b})$ being equal to 0 or 1 . These global flips do change the magnetization in contrast to the above local flips. It should be noted that we do not take the global flips along the real-space direction into account in the present QMC calculation, assuming that their effect is not serious at least when the number $N$ of spins is not too small. This means that we neglect the change of the winding number of the spin configuration. 33 )

Using this approach, we have investigated numerically the ground-state and thermodynamic properties of the spin-1/2-1/2-1-1 chain, confining ourselves to the case where $J_{1}=J_{3}=1.0$ and $J_{2}>0$. (Note that $J_{1}$, or equivalently $J_{3}$, is chosen to be the unit of energy.)

\subsection{Ground-state properties}

In this subsection, we discuss the energy gap and the nearest-neighbor two-spin correlation functions in the ground state, in order to investigate the ground-state phase transition.

For a finite- $N$ system, we have carried out the QMC calculation only with the local flips. Thus, the system is kept in the subspace of a given value of the $z$-component $\mathcal{S}_{\text {tot }}^{z}$ of $\overrightarrow{\mathcal{S}}_{\text {tot }}$. We have estimated the energies $E_{\mathcal{S}_{\text {tot }}^{z}}(N)$ with $\mathcal{S}_{\text {tot }}^{z}=0$ (the ground state) and 1 (the first-excited state), which are expressed as

$$
E_{\mathcal{S}_{\text {tot }}^{z}}(N)=-\left\langle Q_{1}\right\rangle_{\mathrm{MC}, \mathcal{S}_{\text {tot }}^{z}},
$$


where

$$
\begin{aligned}
Q_{1}=\sum_{r=1}^{n} \sum_{\ell=1}^{N / 4}\{ & \left(\frac{\partial \rho_{4 \ell-3,4 \ell-2}^{(2 r-1,2 r)}}{\partial \beta}\right) / \rho_{4 \ell-3,4 \ell-2}^{(2 r-1,2 r)}+\left(\frac{\partial \rho_{4 \ell-1,4 \ell}^{(2 r-1,2 r}}{\partial \beta}\right) / \rho_{4 \ell-1,4 \ell}^{(2 r-1,2 r)} \\
& \left.+\left(\frac{\partial \rho_{4 \ell-2,4 \ell-1}^{(2 r, 2 r+1)}}{\partial \beta}\right) / \rho_{4 \ell-2,4 \ell-1}^{(2 r, 2 r+1)}+\left(\frac{\partial \rho_{4 \ell, 4 \ell+1}^{(2 r, 2 r+1)}}{\partial \beta}\right) / \rho_{4 \ell, 4 \ell+1}^{(2 r, 2 r+1)}\right\}
\end{aligned}
$$

and $\langle\cdots\rangle_{\mathrm{MC}, \mathcal{S}_{\text {tot }}^{z}}$ stands for the Monte Carlo average within the subspace determined by the value of $\mathcal{S}_{\text {tot }}^{z}$. The energy gap $\Delta(N)$ is defined by

$$
\Delta(N)=E_{1}(N)-E_{0}(N)
$$

Carrying out the above-mentioned QMC calculation within the $\mathcal{S}_{\text {tot }}^{z}=0$ subspace, we have also estimated the three kinds of ground-state nearest-neighbor two-spin correlation functions

$$
\begin{gathered}
\omega_{1,2}(N)=\left\langle\vec{s}_{1} \cdot \vec{s}_{2}\right\rangle_{\mathrm{MC}, 0}=3\left\langle s_{1}^{z} s_{2}^{z}\right\rangle_{\mathrm{MC}, 0}, \\
\omega_{2,3}(N)=\left\langle\vec{s}_{2} \cdot \vec{S}_{3}\right\rangle_{\mathrm{MC}, 0}=3\left\langle s_{2}^{z} S_{2}^{z}\right\rangle_{\mathrm{MC}, 0}, \\
\omega_{3,4}(N)=\left\langle\vec{S}_{3} \cdot \vec{S}_{4}\right\rangle_{\mathrm{MC}, 0}=3\left\langle S_{3}^{z} S_{4}^{z}\right\rangle_{\mathrm{MC}, 0} .
\end{gathered}
$$

In these calculations, the values of $J_{2}$ have been chosen to be $J_{2}=1.00,0.90,0.80,0.78,0.75,0.70$, 0.60 , and 0.50 , and those of $N$ to be $N=8,16,32,64$, and 128 . We have performed $10^{6}$ Monte Carlo steps after $10^{5}$ initial steps for obtaining the thermal equilibrium. The Trotter numbers which we have used are $n=12,16,24,32,40$, and 48 , and the $n$-dependence of the finite- $N$ QMC results $A_{n}(N)$ for a given physical quantity $A$ has been extrapolated to $n \rightarrow \infty$ by making a least-squares fit to the formula 35 )

$$
A_{n}(N)=A(N)+\frac{a}{n^{2}}+\frac{b}{n^{4}}
$$

where $a$ and $b$ are constants which are independent of $n ; A(N)$ yields the Trotter-extrapolated value. Most of the calculations have been done at the temperature $k_{\mathrm{B}} T=0.05$ only. For the cases of $J_{2}=1.00$ and 0.50 with $N=8$ and 16 , however, we have also done the calculation at $k_{\mathrm{B}} T=0.02$, and have obtained, for both temperatures, the same Trotter-extrapolated values for $E_{0}(N)$ and $E_{1}(N)$. These values agree with those obtained by the exact-diagonalization calculation within the numerical error, although the conservation of the winding number causes a systematic deviation in short chains. We therefore consider that $k_{\mathrm{B}} T=0.05$ is low enough to discuss the zero-temperature properties. Thus, the calculated result for $E_{0}(N)$ yields the ground-state energy $E_{\mathrm{g}}(N)$ and those for $\omega_{1,2}(N), \omega_{2,3}(N)$, and $\omega_{3,4}(N)$ yield the nearest-neighbor two-spin correlation function in the ground state. 
The Trotter-extrapolated values depend upon which data we use when we make a least-squares fit to the formula given by eq. (3.11). Following Miyashita and Yamamoto's procedure, 36 we have performed three different extrapolations. The first one, denoted by TEX6, uses six data for $n=12$, 16, 24, 32, 40, and 48, the second one, TEX5, uses five data for $n=16,24,32,40$, and 48, and the third one, TEX4, uses four data for $n=24,32,40$, and 48. Both for the ground-state energy $\epsilon_{\mathrm{g}}(N) \equiv E_{0}(N) /(N / 4)$ per unit cell consisting of two $S=1 / 2$ and two $S=1$ spins and for the energy gap $\Delta(N)$, the three extrapolations give almost the same values down to the second decimal place. We determine the Trotter-extrapolated value to be the average of the three values obtained by the three extrapolations, and estimate the error by the difference between the average and the farthest value among the three values. The results for $\epsilon_{\mathrm{g}}(N)$ and $\Delta(N)$ are tabulated, respectively, in Tables II and III, where, for the sake of comparison, the results of the exact-diagonalization calculation for $N=8$ and 16 are also listed.

Table II. Numerical results for the ground-state energy $\epsilon_{\mathrm{g}}(N)$ per unit cell in the case where $J_{1}=J_{3}=1.0$. The values in the columns denoted by TEX give the Totter-extrapolated results, and those in the columns denoted by ED give the results of the exact-diagonalization calculation. The extrapolated values $\epsilon_{\mathrm{g}}(\infty)$ to $N \rightarrow \infty$ are given in the rightmost $(N \rightarrow \infty)$ column. It is noted that the figures in the parentheses show errors in the last digit.

\begin{tabular}{lcccccccc}
\hline & \multicolumn{2}{c}{$N=8$} & \multicolumn{2}{c}{$N=16$} & \multicolumn{2}{c}{$N=32$} & \multicolumn{2}{c}{$N=64$} \\
& TEX & ED & TEX & ED & TEX & TEX & TEX & \\
& & & & & & & \\
\hline$J_{2}=1.00$ & $-3.54(1)$ & -3.5470 & $-3.52(1)$ & -3.5159 & $-3.52(1)$ & $-3.52(1)$ & $-3.52(1)$ & $-3.52(1)$ \\
$J_{2}=0.90$ & $-3.37(1)$ & -3.3832 & $-3.34(1)$ & -3.3449 & $-3.34(1)$ & $-3.34(1)$ & $-3.34(1)$ & $-3.34(1)$ \\
$J_{2}=0.80$ & $-3.22(1)$ & -3.2326 & $-3.19(1)$ & -3.1891 & $-3.18(1)$ & $-3.18(1)$ & $-3.18(1)$ & $-3.18(1)$ \\
$J_{2}=0.78$ & $-3.19(1)$ & -3.2045 & $-3.16(1)$ & -3.1609 & $-3.15(1)$ & $-3.15(1)$ & $-3.15(1)$ & $-3.15(1)$ \\
$J_{2}=0.75$ & $-3.15(1)$ & -3.1638 & $-3.12(1)$ & -3.1209 & $-3.11(1)$ & $-3.11(1)$ & $-3.11(1)$ & $-3.11(1)$ \\
$J_{2}=0.70$ & $-3.09(1)$ & -3.1002 & $-3.06(1)$ & -3.0611 & $-3.05(1)$ & $-3.05(1)$ & $-3.05(1)$ & $-3.05(1)$ \\
$J_{2}=0.60$ & $-2.98(1)$ & -2.9908 & $-2.97(1)$ & -2.9655 & $-2.96(1)$ & $-2.96(1)$ & $-2.96(1)$ & $-2.96(1)$ \\
$J_{2}=0.50$ & $-2.90(1)$ & -2.9060 & $-2.89(1)$ & -2.8937 & $-2.89(1)$ & $-2.89(1)$ & $-2.89(1)$ & $-2.89(1)$ \\
\hline
\end{tabular}

In order to obtain the results in the thermodynamic limit $(N \rightarrow \infty)$, the Trotter-extrapolated values $A(N)$ have further been extrapolated by the least-squares method using a linear function of $1 / N^{2}$,

$$
A(N)=A(\infty)+\frac{c}{N^{2}}
$$


Table III. Numerical results for the energy gap $\Delta(N)$ in the case where $J_{1}=J_{3}=1.0$. The values in the columns denoted by TEX give the Totter-extrapolated results, and those in the columns denoted by ED give the results of the exact-diagonalization calculation. The extrapolated values $\Delta(\infty)$ to $N \rightarrow \infty$ are given in the rightmost $(N \rightarrow \infty)$ column. It is noted that the figures in the parentheses show errors in the last digit.

\begin{tabular}{|c|c|c|c|c|c|c|c|c|}
\hline & \multicolumn{2}{|c|}{$N=8$} & \multicolumn{2}{|c|}{$N=16$} & \multirow{2}{*}{$\begin{array}{c}N=32 \\
\text { TEX }\end{array}$} & \multirow{2}{*}{$\begin{array}{c}N=64 \\
\text { TEX }\end{array}$} & \multirow{2}{*}{$\begin{array}{c}N=128 \\
\text { TEX }\end{array}$} & \multirow[t]{2}{*}{$N \rightarrow \infty$} \\
\hline & TEX & $\mathrm{ED}$ & TEX & ED & & & & \\
\hline$J_{2}=1.00$ & $0.61(1)$ & 0.6156 & $0.46(1)$ & 0.4497 & $0.44(1)$ & $0.44(1)$ & $0.44(1)$ & $0.44(1)$ \\
\hline$J_{2}=0.90$ & $0.55(1)$ & 0.5566 & $0.36(1)$ & 0.3522 & $0.32(1)$ & $0.31(1)$ & $0.31(1)$ & $0.31(1)$ \\
\hline$J_{2}=0.80$ & $0.51(1)$ & 0.5126 & $0.28(1)$ & 0.2807 & $0.17(1)$ & $0.13(1)$ & $0.13(1)$ & $0.13(1)$ \\
\hline$J_{2}=0.78$ & $0.50(1)$ & 0.5064 & $0.27(1)$ & 0.2733 & $0.15(1)$ & $0.09(1)$ & $0.06(2)$ & $0.06(2)$ \\
\hline$J_{2}=0.75$ & $0.50(1)$ & 0.4992 & $0.27(1)$ & 0.2685 & $0.14(1)$ & $0.08(1)$ & $0.06(2)$ & $0.06(2)$ \\
\hline$J_{2}=0.70$ & $0.49(1)$ & 0.4930 & $0.28(1)$ & 0.2785 & $0.20(1)$ & $0.18(1)$ & $0.18(1)$ & $0.18(1)$ \\
\hline$J_{2}=0.60$ & $0.51(1)$ & 0.5059 & $0.37(1)$ & 0.3555 & $0.34(1)$ & $0.34(1)$ & $0.34(1)$ & $0.34(1)$ \\
\hline$J_{2}=0.50$ & $0.56(1)$ & 0.5529 & $0.48(1)$ & 0.4697 & $0.48(1)$ & $0.48(1)$ & $0.48(1)$ & $0.48(1)$ \\
\hline
\end{tabular}

with a constant $c$. Performing the extrapolation, we have used three data $A(32), A(64)$, and $A(128)$ for $J_{2}=0.75$ and 0.78 , and four data $A(16), A(32), A(64)$, and $A(128)$ for the other values of $J_{2}$. Figure 4 illustrate this extrapolation for the energy gap, where $A$ is $\Delta$. The $N \rightarrow \infty$ extrapolated values $\epsilon_{\mathrm{g}}(\infty)$ for the ground-state energy per unit cell and those $\Delta(\infty)$ for the energy gap are also listed in Tables II and III, respectively, and are plotted as a function of $J_{2}$ in Fig. 5 and Fig. 6, respectively. From Fig. 6 we see that $\Delta(\infty)$ vanishes at

$$
J_{2}=J_{2 \mathrm{c}}=0.77 \pm 0.01
$$

This result shows that the ground state of the present system with $J_{1}=J_{3}=1.0$ undergoes a second-order phase transition at this value of $J_{2}$.

The Trotter extrapolated values $\omega_{1,2}(N), \omega_{2,3}(N)$, and $\omega_{3,4}(N)$ for the nearest-neighbor two-spin correlation functions are tabulated in Tables IV, V, and VI, respectively, where the corresponding $N \rightarrow \infty$ extrapolated values, $\omega_{1,2}(\infty), \omega_{2,3}(\infty)$, and $\omega_{3,4}(\infty)$ are also listed. In Fig. 7 we plot $\omega_{1,2}(\infty), \omega_{2,3}(\infty)$, and $\omega_{3,4}(\infty)$ as functions of $J_{2}$, where each correlation function is normalized by its minimum value (note that $-3 / 4 \leq \omega_{1,2}(N) \leq 1 / 4,-1 \leq \omega_{2,3}(N) \leq 1 / 2$, and $-2 \leq \omega_{3,4}(N) \leq 1$ ). This figure demonstrates that as $J_{2}$ increases, $\left|\omega_{1,2}(\infty)\right|$ and $\left|\omega_{2,3}(\infty)\right|$ rapidly decreases and increases, respectively, around $J_{2} \sim J_{2 \mathrm{c}}$, while $\left|\omega_{3,4}(\infty)\right|$ rather gradually decreases with increasing $J_{2}$. Giving a thought to these $J_{2}$-dependences of the correlation functions, we may schematically represent, by means of the VBS picture,20) the ground states for $J_{2}<J_{2 \mathrm{c}}$ and that for $J_{2}>J_{2 \mathrm{c}}$ 
as depicted in Fig. 8. The VBS wave function of Fig. 8(a) corresponds to the state for $J_{2}=0.0$, while the VBS wave function of Fig. $8(\mathrm{~b})$ to the state for $J_{1}=0.0$ and $J_{2} \rightarrow+\infty$. The change of the correlation functions as a function of $J_{2}$ clearly illustrates the interpolation between these two limiting states.

Table IV. Trotter-extrapolated ground-state nearest-neighbor two-spin correlation function $\omega_{1,2}(N)$ and the $N \rightarrow \infty$ extrapolated ground-state nearest-neighbor two-spin correlation function $\omega_{1,2}(\infty)$ in the case where $J_{1}=J_{3}=1.0$. It is noted that the figures in the parentheses show errors in the last digit.

\begin{tabular}{lllllll}
\hline & $N=8$ & $N=16$ & $N=32$ & $N=64$ & $N=128$ & $N \rightarrow \infty$ \\
\hline$J_{2}=1.00$ & $-0.28(1)$ & $-0.23(1)$ & $-0.22(1)$ & $-0.22(1)$ & $-0.22(1)$ & $-0.22(1)$ \\
$J_{2}=0.90$ & $-0.33(1)$ & $-0.27(1)$ & $-0.26(1)$ & $-0.26(1)$ & $-0.26(1)$ & $-0.26(1)$ \\
$J_{2}=0.80$ & $-0.41(1)$ & $-0.37(1)$ & $-0.35(1)$ & $-0.34(1)$ & $-0.34(1)$ & $-0.34(1)$ \\
$J_{2}=0.78$ & $-0.43(1)$ & $-0.40(1)$ & $-0.39(1)$ & $-0.38(1)$ & $-0.38(1)$ & $-0.38(1)$ \\
$J_{2}=0.75$ & $-0.46(1)$ & $-0.46(1)$ & $-0.46(1)$ & $-0.46(1)$ & $-0.46(1)$ & $-0.46(1)$ \\
$J_{2}=0.70$ & $-0.51(1)$ & $-0.53(1)$ & $-0.54(1)$ & $-0.54(1)$ & $-0.54(1)$ & $-0.54(1)$ \\
$J_{2}=0.60$ & $-0.60(1)$ & $-0.62(1)$ & $-0.62(1)$ & $-0.62(1)$ & $-0.62(1)$ & $-0.62(1)$ \\
$J_{2}=0.50$ & $-0.66(1)$ & $-0.67(1)$ & $-0.67(1)$ & $-0.67(1)$ & $-0.67(1)$ & $-0.67(1)$ \\
\hline
\end{tabular}

\subsection{Thermodynamic properties}

We now turn to the discussion of the thermodynamic properties. Performing the QMC calculation which takes into account the global flips given by eqs. (3.5a) and (3.5b) as well as the local flips given by eqs. (3.5a)-(3.5e), we have calculated the temperature dependences of the specific heat $C(N)$ and the magnetic susceptibility $\chi(N)$ for $J_{2}=J_{2 \mathrm{c}}=0.77$, and also for $J_{2}=1.0$ and 5.0. Here, the expressions for $C(N)$ B7 and $\chi(N)$ are given by

$$
C(N)=\frac{1}{k_{\mathrm{B}} T^{2}}\left(\left\langle Q_{1}^{2}\right\rangle_{\mathrm{MC}}-\left\langle Q_{1}\right\rangle_{\mathrm{MC}}^{2}+\left\langle Q_{2}\right\rangle_{\mathrm{MC}}\right)
$$

and

$$
\chi(N)=\frac{1}{T}\left\langle\left(\mathcal{S}_{\text {tot }}^{z}\right)^{2}\right\rangle_{\mathrm{MC}},
$$

where $\langle\cdots\rangle_{\mathrm{MC}}$ denotes the Monte Carlo average, $Q_{1}$ is given by eq. (3.8), and 
Table V. Trotter-extrapolated ground-state nearest-neighbor two-spin correlation function $\omega_{2,3}(N)$ and the $N \rightarrow \infty$ extrapolated ground-state nearest-neighbor two-spin correlation function $\omega_{2,3}(\infty)$ in the case where $J_{1}=J_{3}=1.0$. It is noted that the figures in the parentheses show errors in the last digit.

\begin{tabular}{lllllll}
\hline & $N=8$ & $N=16$ & $N=32$ & $N=64$ & $N=128$ & $N \rightarrow \infty$ \\
\hline$J_{2}=1.00$ & $-0.87(1)$ & $-0.89(1)$ & $-0.89(1)$ & $-0.89(1)$ & $-0.89(1)$ & $-0.89(1)$ \\
$J_{2}=0.90$ & $-0.81(1)$ & $-0.84(1)$ & $-0.85(1)$ & $-0.85(1)$ & $-0.85(1)$ & $-0.85(1)$ \\
$J_{2}=0.80$ & $-0.72(1)$ & $-0.74(1)$ & $-0.75(1)$ & $-0.76(1)$ & $-0.76(1)$ & $-0.76(1)$ \\
$J_{2}=0.78$ & $-0.70(1)$ & $-0.70(1)$ & $-0.71(1)$ & $-0.72(1)$ & $-0.72(1)$ & $-0.72(1)$ \\
$J_{2}=0.75$ & $-0.67(1)$ & $-0.64(1)$ & $-0.63(1)$ & $-0.62(1)$ & $-0.62(1)$ & $-0.62(1)$ \\
$J_{2}=0.70$ & $-0.61(1)$ & $-0.54(1)$ & $-0.52(1)$ & $-0.51(1)$ & $-0.51(1)$ & $-0.51(1)$ \\
$J_{2}=0.60$ & $-0.47(1)$ & $-0.41(1)$ & $-0.40(1)$ & $-0.40(1)$ & $-0.40(1)$ & $-0.40(1)$ \\
$J_{2}=0.50$ & $-0.35(1)$ & $-0.31(1)$ & $-0.31(1)$ & $-0.31(1)$ & $-0.31(1)$ & $-0.31(1)$ \\
\hline
\end{tabular}

Table VI. Trotter-extrapolated ground-state nearest-neighbor two-spin correlation function $\omega_{3,4}(N)$ and the $N \rightarrow \infty$ extrapolated ground-state nearest-neighbor two-spin correlation function $\omega_{3,4}(\infty)$ in the case where $J_{1}=J_{3}=1.0$. It is noted that the figures in the parentheses show errors in the last digit.

\begin{tabular}{lllllll}
\hline & $N=8$ & $N=16$ & $N=32$ & $N=64$ & $N=128$ & $N \rightarrow \infty$ \\
\hline$J_{2}=1.00$ & $-1.58(1)$ & $-1.53(1)$ & $-1.52(1)$ & $-1.52(1)$ & $-1.52(1)$ & $-1.52(1)$ \\
$J_{2}=0.90$ & $-1.62(1)$ & $-1.57(1)$ & $-1.56(1)$ & $-1.56(1)$ & $-1.56(1)$ & $-1.56(1)$ \\
$J_{2}=0.80$ & $-1.69(1)$ & $-1.66(1)$ & $-1.64(1)$ & $-1.63(1)$ & $-1.63(1)$ & $-1.63(1)$ \\
$J_{2}=0.78$ & $-1.70(1)$ & $-1.68(1)$ & $-1.67(1)$ & $-1.66(1)$ & $-1.66(1)$ & $-1.66(1)$ \\
$J_{2}=0.75$ & $-1.73(1)$ & $-1.73(1)$ & $-1.72(1)$ & $-1.72(1)$ & $-1.72(1)$ & $-1.72(1)$ \\
$J_{2}=0.70$ & $-1.78(1)$ & $-1.79(1)$ & $-1.80(1)$ & $-1.80(1)$ & $-1.80(1)$ & $-1.80(1)$ \\
$J_{2}=0.60$ & $-1.86(1)$ & $-1.87(1)$ & $-1.87(1)$ & $-1.87(1)$ & $-1.87(1)$ & $-1.87(1)$ \\
$J_{2}=0.50$ & $-1.92(1)$ & $-1.92(1)$ & $-1.92(1)$ & $-1.92(1)$ & $-1.92(1)$ & $-1.92(1)$ \\
\hline
\end{tabular}

$$
Q_{2}=\sum_{r=1}^{n} \sum_{\ell=1}^{N / 4}\left[\left(\frac{\partial^{2} \rho_{4 \ell-3,4 \ell-2}^{(2 r-1,2 r)}}{\partial \beta^{2}}\right) / \rho_{4 \ell-3,4 \ell-2}^{(2 r-1,2 r)}-\left\{\left(\frac{\partial \rho_{4 \ell-3,4 \ell-2}^{(2 r-1,2 r)}}{\partial \beta}\right) / \rho_{4 \ell-3,4 \ell-2}^{(2 r-1,2 r)}\right\}^{2}\right.
$$




$$
\begin{aligned}
& +\left(\frac{\partial^{2} \rho_{4 \ell-1,4 \ell}^{(2 r-1,2 r)}}{\partial \beta^{2}}\right) / \rho_{4 \ell-1,4 \ell}^{(2 r-1,2 r)}-\left\{\left(\frac{\partial \rho_{4 \ell-1,4 \ell}^{(2 r-1,2 r)}}{\partial \beta}\right) / \rho_{4 \ell-1,4 \ell}^{(2 r-1,2 r)}\right\}^{2} \\
& +\left(\frac{\partial^{2} \rho_{4 \ell-2,4 \ell-1}^{(2 r, 2 r+1)}}{\partial \beta^{2}}\right) / \rho_{4 \ell-2,4 \ell-1}^{(2 r, 2 r+1)}-\left\{\left(\frac{\partial \rho_{4 \ell-2,4 \ell-1}^{(2 r, 2 r+1)}}{\partial \beta}\right) / \rho_{4 \ell-2,4 \ell-1}^{(2 r, 2 r+1)}\right\}^{2} \\
& \left.+\left(\frac{\partial^{2} \rho_{4 \ell, 4 \ell+1}^{(2 r, 2 r+1)}}{\partial \beta^{2}}\right) / \rho_{4 \ell, 4 \ell+1}^{(2 r, 2 r+1)}-\left\{\left(\frac{\partial \rho_{4 \ell, 4 \ell+1}^{(2 r, 2 r+1)}}{\partial \beta}\right) / \rho_{4 \ell, 4 \ell+1}^{(2 r, 2 r+1)}\right\}^{2}\right] .
\end{aligned}
$$

In deriving eq. (3.15), we assume that the g-factor associated with the $S=1 / 2$ spin is identical to that associated with the $S=1$ spin and that $\left\langle\mathcal{S}_{\text {tot }}^{z}\right\rangle_{\mathrm{MC}}$ vanishes. For $J_{2}=1.0$ and 5.0 , the values of $N$ have been chosen only to be $N=8,16$ and 32 . This is because for these $J_{2}$ 's, we expect weak $N$-dependences of $C(N) /(N / 4)$ and $\chi(N) /(N / 4)$, since the energy gap $\Delta(\infty)$ is rather large (see Fig. 6), or equivalently, the correlation length is rather short. For the critical value $J_{2}=0.77$, on the other hand, the calculation has been done also for $N=64$ and 128 when $T$ is not too high, in addition to $N=8,16$ and 32 . We list in Table VII Trotter numbers $n^{\prime} s$, the Monte Carlo steps, and the initial steps for obtaining the thermal equilibrium. By using the QMC results for all of the four Trotter numbers, the $n$-dependence of $C_{n}(N)$ for $J_{2}=0.77$ and 1.0 has been extrapolated to $n \rightarrow \infty$ by the least-squares method with the formula of eq. (3.11) Similar extrapolations for $C_{n}(N)$ for $J_{2}=5.0$ and for $\chi_{n}(N)$ for $J_{2}=0.77,1.0$, and 5.0 have been done by the use of the formula 35 .

$$
A_{n}(N)=A(N)+\frac{a}{n^{2}}
$$

where $a$ is a constant, instead of the formula of eq. (3.11).

In Fig. 9 we plot versus the temperature $T$ the Trotter-extrapolated values $C(N) /(N / 4)$ [Fig. 9(a)] with $N=8,16$, and 32 for the specific heat per unit cell and $\chi(N) /(N / 4)$ [Fig. 9(b)] with $N=8$, 16 , and 32 for the magnetic susceptibility per unit cell, which have been obtained for $J_{2}=1.0$. For the sake of comparison, we also plot, in these figures, the exact results for both quantities for $N=8$ and $J_{2}=1.0$ versus $T$, which we have obtained by diagonalizing completely the Hamiltonian to calculate all the eigenvalues. The corresponding plots for $J_{2}=0.77$ are presented in Fig. 10 . As is expected, both $C(N) /(N / 4)$ and $\chi(N) /(N / 4)$ for $J_{2}=1.0$ have rather weak $N$-dependences even at $k_{\mathrm{B}} T=0.08$. For $J_{2}=0.77$, on the other hand, the $N$-dependence of $\chi(N) /(N / 4)$ is noticeable at low temperatures; it increases as $N$ increases. Judging from the $T$-dependences of $\chi(64) / 16$ and $\chi(128) / 32$, we may conclude that at $T=0.0$, the magnetic susceptibility per unit cell in the thermodynamic limit is finite for $J_{2}=0.77$ with the value $[\chi(N) /(N / 4)]_{N \rightarrow \infty}=0.42 \pm 0.01$. This is consistent with the fact that the energy gap vanishes for this value of $J_{2}$. 
Table VII. Sets of the Trotter numbers (n) used at various temperatures, and also the Monte Carlo steps (MCS) and the initial steps (IS) spent for each Trotter number at each temperature, where the former steps do not include the latter steps. Note that $J_{1}=J_{3}=1.0$.

\begin{tabular}{ccccc}
\hline$k_{\mathrm{B}} T$ for $J_{2}=0.77,1.0$ & $k_{\mathrm{B}} T$ for $J_{2}=5.0$ & $n$ & MCS & IS \\
\hline $0.08 \leq k_{\mathrm{B}} T \leq 0.10$ & $0.400 \leq k_{\mathrm{B}} T \leq 0.625$ & $12,16,24,32$ & $120 \times 10^{4}$ & $6 \times 10^{4}$ \\
$k_{\mathrm{B}} T=0.15$ & $k_{\mathrm{B}} T=0.750$ & $8,12,16,24$ & $100 \times 10^{4}$ & $5 \times 10^{4}$ \\
& $k_{\mathrm{B}} T=0.875$ & $8,12,16,24$ & $50 \times 10^{4}$ & $5 \times 10^{4}$ \\
$k_{\mathrm{B}} T=0.20$ & $1.000 \leq k_{\mathrm{B}} T \leq 1.375$ & $6,8,12,16$ & $50 \times 10^{4}$ & $5 \times 10^{4}$ \\
$0.30 \leq k_{\mathrm{B}} T \leq 0.50$ & $1.500 \leq k_{\mathrm{B}} T \leq 2.750$ & $4,6,8,12$ & $40 \times 10^{4}$ & $5 \times 10^{4}$ \\
$0.60 \leq k_{\mathrm{B}} T \leq 1.00$ & $3.000 \leq k_{\mathrm{B}} T \leq 5.000$ & $2,4,6,8$ & $30 \times 10^{4}$ & $5 \times 10^{4}$ \\
$1.20 \leq k_{\mathrm{B}} T \leq 2.00$ & $6.000 \leq k_{\mathrm{B}} T \leq 8.000$ & $2,4,6,8$ & $25 \times 10^{4}$ & $5 \times 10^{4}$ \\
\hline
\end{tabular}

Figure 11 presents the plots versus $T$ of $C(N) /(N / 4)$ [Fig. 11(a)] with $N=8,16$, and 32 and $\chi(N) /(N / 4)$ [Fig. 11(b)] with $N=8,16$, and 32, which have been obtained for $J_{2}=5.0$, and also the plots versus $T$ of the exact results for both quantities for $N=8$ and $J_{2}=5.0$. It is noted that the $N$-dependences of both $C(N) /(N / 4)$ and $\chi(N) /(N / 4)$ are weak as in the case of $J_{2}=1.0$. A characteristic feature of the $C(N) /(N / 4)$ versus $T$ curve shown in Fig. 11(a) is that it has a double peak. The origin of the two peaks can be understood as follows. The dot-dashed line in Fig. 11(a) shows the $T$-dependence of the specific heat for two pairs of the two-spin system consisting of an $S=1 / 2$ spin $\vec{s}$ and an $S=1$ spin $\vec{S}$ which couple with each other by $5 \vec{s} \cdot \vec{S}$. Comparing this with the $T$-dependence of $C(N) /(N / 4)$, we may conclude that the higher-temperature peak is associated with the Schottky-type peak of this two-spin system. On the other hand, the dashed line in Fig. 11(a) shows the $T$-dependence of the half of the specific heat for the $S=1 / 2$ bond-alternating chain described by the Hamiltonian $\mathcal{H}_{(a)}$ [see eq. (2.3)] with $J_{1}=J_{3}=1.0$ and $N=8$. The peak height as well as the peak position in this $T$-dependence agrees, respectively, with those in the $T$-dependence of $C(N) /(N / 4)$, which may leads us to the conclusion that the lower-temperature peak is associated with the peak coming from the short-range order in the $S=1 / 2$ bond-alternating chain. A somewhat unusual behavior of $\chi(N) /(N / 4)$ as a function of $T$, seen in Fig. 11(b), can also be understood as follows. At high temperatures the $T$-dependence of $\chi(N) /(N / 4)$ should be similar to that of the magnetic susceptibility for two pairs of the above two-spin system, which diverges at $T \rightarrow 0.0$ [see the dot-dashed line in Fig. 11(b)], while $\chi(N) /(N / 4)$ should vanish at $T=0.0$ since the present system has massive excitations when $J_{2}=5.0$. 


\section{$\S 4 . \quad$ Summary and Conclusions}

We have explored the ground-state properties of the quantum mixed spin system described by the Hamiltonian $\mathcal{H}[$ see eq. (1.1)]. First, we have applied the Lieb-Mattis theorem 17 to show that the ground state of the system is nonmagnetic when $J_{1}>0$ and when $J_{1}<0$ and $J_{3}>0$; in the remaining region where $J_{1}<0$ and $J_{3}<0$ it is ferrimagnetic or ferromagnetic depending upon whether $J_{2}>0$ or $J_{2}<0[$ Table I]. Second, applying the Wigner-Eckart theorem 19 ) to the limiting cases (a) $\left|J_{2}\right| \gg\left|J_{1}\right|,\left|J_{3}\right|$ with $J_{2}>0$, (b) $\left|J_{2}\right| \gg\left|J_{1}\right|,\left|J_{3}\right|$ with $J_{2}<0$, and (c) $\left|J_{1}\right| \gg\left|J_{2}\right|,\left|J_{3}\right|$ with $J_{1}<0$, we have mapped the Hamiltonian $\mathcal{H}$ into the bond-alternating chains described by the Hamiltonians $\mathcal{H}_{(a)}$ with effective $S=1 / 2$ spins $[$ eq. $(2.3)], \mathcal{H}_{(b)}$ with effective $S=3 / 2$ spins $[$ eq. $(2.5)]$, and $\mathcal{H}_{(c)}$ with effective $S=1$ spins [eq. (2.7)], respectively. This leads to the facts that in the limiting case (a) the present system is massless when eq. (2.8) holds, and in the limiting case (b) it is massless when eq. (2.9) holds. We have also carried out the perturbation calculations to show that in the limiting cases (d) $J_{3} \gg\left|J_{1}\right|,\left|J_{2}\right|$ and (e) $J_{1} \gg\left|J_{2}\right|$ and $J_{1} \gg J_{3}>0$, the system is massless when eqs. (2.14) and (2.18) hold, respectively. These results for $J_{3}=1.0$ are summarized in Fig. 2. Third, performing the QMC calculation without the global flips at a sufficiently low temperature $\left(k_{\mathrm{B}} T=0.05\right)$, we have shown that when $J_{1}=J_{3}=1.0$, the energy gap $\Delta(\infty)$ vanishes at $J_{2}=J_{2 \mathrm{c}}=0.77 \pm 0.01$ [Fig. 6], where the second-order phase transition occurs in the ground state. The representation of both the ground state for $J_{2}<J_{2 \mathrm{c}}$ and that for $J_{2}>J_{2 \mathrm{c}}$ by means of the VBS picture is given in Fig. 8, which is suggested from the $J_{2}$ dependences [Fig. 7] of the ground-state nearest-neighbor two-spin correlation functions $\omega_{1,2}(\infty), \omega_{2,3}(\infty)$, and $\omega_{3,4}(\infty)$.

Furthermore, performing the QMC calculation including the global flips along the Trotter direction as well as the local flips, we have calculated the temperature dependences of the specific heat $C(N) /(N / 4)$ per unit cell and the magnetic susceptibility $\chi(N) /(N / 4)$ per unit cell with $N=8$, 16 , and 32 for $J_{2}=0.77,1.0$, and 5.0 with $J_{1}$ and $J_{3}$ fixed at $J_{1}=J_{3}=1.0$. The results of these calculations are depicted in Figs. 9-11.

Figure 2 clearly demonstrates that several massless lines exist on the $J_{1}$ versus $J_{2}$ plane with $J_{1}>0$ in the case of $J_{3}=1.0$. We are now exploring the details of the ground-state phase diagram on this plane by the use of a density-matrix renormalization-group method proposed originally by White 38) Our preliminary results show that there exist four massless lines which divides the upper-half plane into six regions; the ground state in each region can be understood by the VBS picture.31)

In conclusion, we hope that the present study stimulates further experimental studies on related subject, which include the synthesization of quantum mixed spin systems with nonmagnetic ground states. 


\section{Acknowledgements}

We would like to thank Professor S. Yamamoto, Dr. T. Fukui, and Dr. K. Totsuka for invaluable discussions. We also thank the Supercomputer Center, Institute for Solid State Physics, University of Tokyo and the Computer Center, Tohoku University for computational facilities. One of us (H.J. M.) gratefully acknowledges the financial support from the Japan Society for the Promotion of Science. The present work has been supported in part by a Grant-in-Aid for Scientific Research (C) and for International Scientific Research (Joint Research) from the Ministry of Education, Science, Sports and Culture.

[1] F. D. M. Haldane: Phys. Lett. 93A (1983) 464; F. D. M. Haldane: Phys. Rev. Lett. 50 (1983) 1153.

[2] For reviews, see I. Affleck: J. Phys.: Condens. Matter 1 (1989) 3047; M. Takahashi and T. Sakai: Computational Physics as a New Frontier in Condensed Matter Research, ed. H. Takayama, M. Tsukada, H. Shiba, F. Yonezawa, M. Imada and Y. Okabe (Physical Society of Japan, Tokyo, 1995) p. 180.

[3] O. Kahn: Magnetism of the Heteropolymetallic Systems, Structure and Bonding, Vol. 68 (Springer-Verlag, Berlin, 1987) p. 89, and references therein; O. Kahn, Y. Pei and Y. Journaux: Inorganic Materials, ed. D. W. Bruce and D. O'Hare (John Wiley \& Sons, Chichester, 1996) p. 65, and reference therein; O. Kahn: Molecular Magnetism (VCH, New York, 1993), and references therein; O. Kahn: Adv. Inorg. Chem. 43 (1995) 179, and reference therein.

[4] Y. Pei, M. Verdaguer, O. Kahn, J. Sletten and J.-P. Renard: Inorg. Chem. 26 (1987) 138.

[5] H. J. de Vega and F. Woynarovich: J. Phys. A 25 (1992) 4499; H. J. de Vega, L. Mezincescu and R. I. Nepomechie: Phys. Rev. B 49 (1994) 13223; H. J. de Vega, L. Mezincescu and R. I. Nepomechie: Int. J. Mod. Phys. B 8 (1994) 3473.

[6] S. R. Aladim and M. J. Martins: J. Phys. A 26 (1993) L529; M. J. Martins: J. Phys. A 26 (1993) 7301.

[7] M. Fujui, S. Fujimoto and N. Kawakami: J. Phys. Soc. Jpn. 65 (1996) 2381.

[8] St. Meißner and B.-D. Dörfel: J. Phys. A 29 (1996) 1949; B.-D. Dörfel and St. Meißner: J. Phys. A 29 (1996) 6471; B.-D. Dörfel and St. Meißner: J. Phys. A 30 (1997) 1831; B.-D. Dörfel and St. Meißner: to be published in J. Phys. A: Math. Gen. (hep-th/9708107).

[9] A. K. Kolezhuk, H.-J. Mikeska and S. Yamamoto: Phys. Rev. B 55 (1997) R3336.

[10] S. Brehmer, H.-J. Mikeska and S. Yamamoto: J. Phys.: Condens. Matter 9 (1997) 3921.

[11] S. K. Pati, S. Ramasesha and D. Sen: Phys. Rev. B 55 (1997) 8894; S. K. Pati, S. Ramasesha and D. Sen: J. Phys.: Condens. Matter 9 (1997) 8707.

[12] F. C. Alcaraz and A. L. Malvezzi: J. Phys. A: Math. Gen. 30 (1997) 767.

[13] H. Niggemann, G. Uimin and J. Zittartz: J. Phys.: Condens. Matter 9 (1997) 9031.

[14] T. Fukui and N. Kawakami: Phys. Rev. B 55 (1997) R14709; T. Fukui and N. Kawakami: Phys. Rev. B 56 (1997) 8799.

[15] S. Yamamoto: Int. J. Mod. Phys. C 8 (1977) 609.

[16] T. Morita, M. Katsumura, T. Nishimura, M. Sugimoto, T. Kuramoto and T. Ono: private communication.

[17] E. Lieb and D. Mattis: J. Math. Phys. 3 (1962) 749.

[18] A preliminary report of the present study will be published by T. Tonegawa, T. Hikihara, T. Nishino, M. Kaburagi, S. Miyashita and H.-J. Mikeska: Proc. Int. Conf. on Magnetism, Cairns, 1997, J. Magn. Magn. Mater. 
[19] See, for example, J. J. Sakurai, Modern Quantum Mechanics (Benjamin/Cummings, Menlo Park, 1985 ) p. 238.

[20] I. Affleck, T. Kennedy, E. H. Lieb and H. Tasaki: Phys. Rev. Lett. 59 (1987) 799; I. Affleck, T. Kennedy, E. H. Lieb and H. Tasaki: Commun. Math. Phys. 115 (1988) 477.

[21] See, for example, J. W. Bray, L. V. Interrante, I. S. Jacobs and J. C. Bonner: Extended Linear Chain Compounds, ed. J. S. Miller (Plenum, New York, 1982) Vol. 3, p. 353.

[22] I. Affleck: Nucl. Phys. B 257 (1985) 397; Nucl. Phys. B 265 (1986) 409; I. Affleck and F. D. M. Haldane: Phys. Rev. B 36 (1987) 5291.

[23] S. Yamamoto: Phys. Rev. B 52 (1995) 10170.

[24] M. Yajima and M. Takahashi: J. Phys. Soc. Jpn. 65 (1996) 39.

[25] S. Yamamoto: Phys. Rev. B 55 (1997) 3603.

[26] K. Okamoto and K. Nomura: Phys. Lett. A 169 (1992) 433; G. Castilla, S. Chakravarty and V. J. Emery: Phys. Rev. Lett. 75 (1995) 1823; S. Eggert: Phys. Rev. B 54 (1996) R9612.

[27] R. R. P. Singh and M. P. Gelfand: Phys. Rev. Lett. 61 (1988) 2133.

[28] Y. Kato and A. Tanaka: J. Phys. Soc. Jpn. 63 (1994) 1277.

[29] S. Yamamoto: J. Phys. Soc. Jpn. 63 (1994) 4327.

[30] K. Totsuka, Y. Nishiyama, N. Hatano and M. Suzuki: J. Phys.: Condens. Matter 7 (1995) 4895.

[31] T. Hikihara, T. Tonegawa, M. Kaburagi, T. Nishino, S. Miyashita and H.-J. Mikeska: in preparation.

[32] M. Suzuki: Prog. Theor. Phys. B 56 (1976) 1454.

[33] J. E. Hirsch, R. L. Sugar, D. J. Scalapino and R. Blankenbecler: Phys. Rev. B 26 (1982) 5033.

[34] See, for example, S. Miyashita and S. Yamamoto: Phys. Rev. B 48 (1993) 913; S. Yamamoto and S. Miyashita: Phys. Rev. B 50 (1994) 6277; S. Yamamoto: Phys. Rev. B 53 (1996) 3364.

[35] M. Suzuki: Quantum Monte Carlo Methods in Equilibrium and Nonequilibrium Systems, ed. M. Suzuki (SpringerVerlag, Belrin, 1987) p. 2.

[36] The first paper of ref. 34 .

[37] M. Suzuki, S. Miyashita and A. Kuroda: Prog. Theor. Phys. 58 (1977) 1377.

[38] S. R. White: Phys. Rev. Lett. 69 (1992) 2863; S. R. White: Phys. Rev. B 48 (1993) 10345. 
Fig. 1. Unit cells of the lowest-energy spin arrays, for (a) $J_{3}>0$ and (b) $J_{3}<0$, of the corresponding classical system in which $\vec{s}_{\ell}$ and $\vec{S}_{\ell}$ are replaced, respectively, by the classical spin vectors with the magnitudes of $1 / 2$ and 1 . The short and long arrows indicate the former and latter classical spin vectors, respectively.

Fig. 2. Massless lines in the $J_{1}$ versus $J_{2}$ plane with $J_{1}>0$ and $J_{3}=1.0$ in the limiting cases (a), (b), (d), and (e). The massless lines in the cases (a), (b), and (e) are shown by the dashes lines, while the massless line in the case (d) is by the solid line in the box where the scales of both the abscissa and the ordinate are enlarged by factor 10. The dotted lines show simple extrapolations of the solid line. The cirle denotes the massless point $\left(J_{2}=0.77\right.$, $\left.J_{1}=1.0\right)$, which is obtained in $\S 3.2$ [see eq. (3.13)].

Fig. 3. A graphical representation of the two-dimensional Ising system, where the horizontal and vertical directions correspond, respectively, the real-space and Trotter directions. The open circles denote the Ising variables $\left\{s_{4 \ell-3}^{\left(r^{\prime}\right)}\right\}$ and $\left\{s_{4 \ell-2}^{\left(r^{\prime}\right)}\right\}$, which take the two values $\pm \frac{1}{2}$, and the open squares denote the Ising variables $\left\{S_{4 \ell-1}^{\left(r^{\prime}\right)}\right\}$, and $\left\{S_{4 \ell}^{\left(r^{\prime}\right)}\right\}$, which take the three values 0 and \pm 1 . The plaquettes shaded by the slashed, back-slashed, vertical, and horizontal lines correspond, respectively, to the local Boltzmann factors, $\rho_{4 \ell-3,4 \ell-2}^{(2 r-1,2 r)}, \rho_{4 \ell-1,4 \ell}^{(2 r-1,2 r)}, \rho_{4 \ell-2,4 \ell-1}^{(2 r, 2 r+1)}$, and $\rho_{4 \ell, 4 \ell+1}^{(2 r, 2 r+1)}$.

Fig. 4. Plot of the Trotter-extrapolated energy gap $\Delta(N)$ versus $1 / N$ with $N=16,32,64$, and 128 , for (a) $J_{2}=1.00$ (the open circles), $J_{2}=0.90$ (the open squares), $J_{2}=0.80$ (the open diamonds), and $J_{2}=0.78$ (the open triangles) and for (b) $J_{2}=0.75$ (the closed triangles), $J_{2}=0.70$ (the closed diamonds), $J_{2}=0.60$ (the closed squares), and $J_{2}=0.50$ (the closed circles), where $J_{1}=J_{3}=1.0$. The solid lines give curves of a least-squares fit to eq. (3.12) (see the text for more details). 
Fig. 5. Plot versus $J_{2}$ of the $N \rightarrow \infty$ extrapolated ground-state energy per unit cell $\epsilon_{\mathrm{g}}(\infty)$, where $J_{1}=J_{3}=1.0$. The solid line is a guide to the eye.

Fig. 6. Plot versus $J_{2}$ of the $N \rightarrow \infty$ extrapolated energy gap $\Delta(\infty)$, where $J_{1}=J_{3}=1.0$. The solid lines are guides to the eye.

Fig. 7. Plots versus $J_{2}$ of the $N \rightarrow \infty$ extrapolated ground-state nearest-neighbor two-spin correlation functions $\omega_{1,2}(\infty)$ (the open circles), $\omega_{2,3}(\infty)$ (the open squares), and $\omega_{3,4}(\infty)$ (the open diamonds), each of which is normalized by its minimum value. Note that $J_{1}=J_{3}=1.0$. The solid lines are guides to the eye.

Fig. 8. Schematic representations of the ground states for (a) $J_{2}<J_{2 \mathrm{c}}$ and (b) $J_{2}>J_{2 \mathrm{c}}$ by means of the VBS picture,20 where $J_{1}=J_{3}=1.0$. The solid circles represent the $S=1 / 2$ spins, and two $S=1 / 2$ spins connected by the solid line form a singlet pair. Each open ellipse surrounding two $S=1 / 2$ represents an operation of constructing an $S=1$ spin from these $S=1 / 2$ spins by symmetrizing them.

Fig. 9. Plot versus the temperature $T$ of (a) the Trotter-extrapolated specific heat $C(N) /(N / 4)$ per unit cell and (b) the Trotter-extrapolated magnetic susceptibility $\chi(N) /(N / 4)$ per unit cell with $N=8$ (the open circles), 16 (the crosses), and 32 (the open diamonds) for $J_{2}=1.0$. The dotted lines in (a) and (b) show, respectively, the exact results for the specific heat per unit cell and the magnetic susceptibility per unit cell, for $N=8$ and $J_{2}=1.0$. Note that $J_{1}=J_{3}=1.0$. 
Fig. 10. Plot versus the temperature $T$ of (a) the Trotter-extrapolated specific heat $C(N) /(N / 4)$ per unit cell and (b) the Trotter-extrapolated magnetic susceptibility $\chi(N) /(N / 4)$ per unit cell with $N=8$ (the open circles), 16 (the crosses), and 32 (the open diamonds), 64 (the open squares), and 128 (the open triangles) for $J_{2}=0.77$, being the critical value of $J_{2}$. Here, $C(N)$ and $\chi(N)$ with $N=64$ and 128 are given only when $k_{\mathrm{B}} T \leq 0.50$. The dotted lines in (a) and (b) show, respectively, the exact results for the specific heat per unit cell and the magnetic susceptibility per unit cell, for $N=8$ and $J_{2}=0.77$. Note that $J_{1}=J_{3}=1.0$.

Fig. 11. Plot versus the temperature $T$ of (a) the Trotter-extrapolated specific heat $C(N) /(N / 4)$ per unit cell and (b) the Trotter-extrapolated magnetic susceptibility $\chi(N) /(N / 4)$ per unit cell with $N=8$ (the open circles), 16 (the crosses), and 32 (the open diamonds) for $J_{2}=5.0$. The dotted lines in (a) and (b) show, respectively, the exact results for the specific heat per unit cell and the magnetic susceptibility per unit cell, for $N=8$ and $J_{2}=5.0$. Note that $J_{1}=J_{3}=1.0$. The dot-dashed lines in (a) and (b) show, respectively, the specific heat and the magnetic susceptibility for two pairs of the two-spin system consisting of an $S=1 / 2$ spin $\vec{s}$ and an $S=1$ spin $\vec{S}$ which couple with each other by $5 \vec{s} \cdot \vec{S}$. Furthermore, the dashed line in (a) shows the half of the specific heat for the $S=1 / 2$ bond-alternating chain described by the Hamiltonian $\mathcal{H}_{(a)}$ given by eq. (2.3) with $J_{1}=J_{3}=1.0$ and $N=8$. 
This figure "fig01a.jpg" is available in "jpg" format from: http://arxiv.org/ps/cond-mat/9712296v2 
This figure "fig01b.jpg" is available in "jpg" format from: http://arxiv.org/ps/cond-mat/9712296v2 
This figure "fig02.jpg" is available in "jpg" format from: http://arxiv.org/ps/cond-mat/9712296v2 
This figure "fig03.jpg" is available in "jpg" format from: http://arxiv.org/ps/cond-mat/9712296v2 
This figure "fig04a.jpg" is available in "jpg" format from: http://arxiv.org/ps/cond-mat/9712296v2 
This figure "fig04b.jpg" is available in "jpg" format from: http://arxiv.org/ps/cond-mat/9712296v2 
This figure "fig05.jpg" is available in "jpg" format from: http://arxiv.org/ps/cond-mat/9712296v2 
This figure "fig06.jpg" is available in "jpg" format from: http://arxiv.org/ps/cond-mat/9712296v2 
This figure "fig07.jpg" is available in "jpg" format from: http://arxiv.org/ps/cond-mat/9712296v2 
This figure "fig08.jpg" is available in "jpg" format from: http://arxiv.org/ps/cond-mat/9712296v2 
This figure "fig09a.jpg" is available in "jpg" format from: http://arxiv.org/ps/cond-mat/9712296v2 
This figure "fig09b.jpg" is available in "jpg" format from: http://arxiv.org/ps/cond-mat/9712296v2 
This figure "fig10a.jpg" is available in "jpg" format from: http://arxiv.org/ps/cond-mat/9712296v2 
This figure "fig10b.jpg" is available in "jpg" format from: http://arxiv.org/ps/cond-mat/9712296v2 
This figure "fig11a.jpg" is available in "jpg" format from: http://arxiv.org/ps/cond-mat/9712296v2 
This figure "fig11b.jpg" is available in "jpg" format from: http://arxiv.org/ps/cond-mat/9712296v2 